\title{
FoxO3a mediates glioma cell invasion by regulating MMP9 expression
}

\author{
$\mathrm{KE} \mathrm{XU}^{1 *}$, HUA PEI $^{1}$, ZHENHAO ZHANG $^{2 *}$, SUFANG DONG ${ }^{3}$, \\ RUI-JIA FU ${ }^{3}$, WEN-MING WANG ${ }^{3}$ and HUAMIN WANG ${ }^{1}$
}

\author{
${ }^{1}$ Department of Immunology, School of Tropical and Laboratory Medicine, Hainan Medical University, \\ Haikou, Hainan 571101; ${ }^{2}$ Medical Technology Institute of Xuzhou Medical College, Xuzhou, Jiangsu 221004; \\ ${ }^{3}$ School of Tropical and Laboratory Medicine, Hainan Medical University, Haikou, Hainan 571101, P.R. China
}

Received February 23, 2016; Accepted August 8, 2016

DOI: $10.3892 / o r .2016 .5087$

\begin{abstract}
The role of FoxO3a in glioma progression is poorly defined. Herein, we show that silencing FoxO3a in U251 cells leads to decreased invasive migration and proliferation, while ectopic expression of FoxO3a in U87 cells with weak endogenous FoxO3a protein levels enhances invasion and proliferation. To further investigate the mechanism by which FoxO3a promotes invasion, we detected key members of the matrix metalloproteinases (MMPs) that are associated with invasion. Our findings showed that, among these MMP members, only FoxO3a induced MMP9 expression, and MMP9 overexpression reversed the effect that silencing FoxO3a had on the attenuation of cell invasion. Taken together, these data link FoxO3a to the promotion of metastasis in glioma cells.
\end{abstract}

\section{Introduction}

Glioblastoma multiforme (GBM) is the most common primary malignant brain tumor in adults. Patients with GBM have a poor prognosis, and have a median survival of 1 year despite aggressive therapy; fewer than 5\% survive for 5 years (1). One reason for this poor prognosis is that GBM has the ability to infiltrate and invade the surrounding normal brain tissue and thereby avoid surgical and radiological interventions. These residual metastatic cancer cells contribute to recurrence, leading to the fatal outcome of this disease (2). While invading through these spaces, glioma cells typically undergo several biological changes, including an increase in their ability to degrade the extracellular matrix (ECM) (3). Many studies have implicated matrix-metalloproteinases (MMPs) in the

Correspondence to: Professor Huamin Wang, Department of Immunology, School of Tropical and Laboratory Medicine, Hainan Medical University, Haikou, Hainan 571101, P.R. China

E-mail: alan-dd-shore@hotmail.com

*Contributed equally

Key words: FoxO3a, matrix metalloproteinase 9, U251, U87 degradation of the ECM, and increased expression of several MMPs occurs in cancer cells compared with their normal cell counterparts, including glioma cells (4).

Forkhead box O (FOXO) transcription factors have critical roles in a number of physiological and pathological processes. In mammals, four subfamily members have been identified, FoxO1, FoxO3, FoxO4, and FoxO6 (5). Of the Forkhead transcription factors, FoxO3a is the member that has emerged as a versatile target for multiple disorders, including various tumors. Hu and colleagues have shown that FoxO3a is linked to the poor survival of patients with breast tumors (6). In addition to breast cancer (7-9), FoxO3a has been shown to be involved in several other tumor types, including prostate cancer, glioblastoma and leukemia (10-15). The role of FoxO3a in cancer progression is primarily attributed to the two most significant cellular processes regulated by FoxO3a: i) FoxO3a activation increases the levels of cell cycle inhibitor proteins p21 and p27, both of which subsequently suppress the G1 to $\mathrm{S}$ cell cycle transition (16-18), and ii) the expression of FoxO3a leads to pro-apoptosis $(19,20)$ through the modulation of the expression of its target genes. For example, FoxO3A induces tumor necrosis factor-related apoptosis-induced ligand (TRAIL) and the BH3-only proteins Noxa and Bim (21).

Prior studies advocated that FoxO3a functions as a tumor suppressor and that it is likely to be an important target for the inhibition of cancer cell progression. However, recent studies highlight the opposing functions of the protein. In invasive ductal breast carcinoma, FoxO3a is linked to lymph node metastasis and poor survival, and its activation does not induce cell death or cell cycle arrest (8). The overexpression of FoxO3a in the MDA-MB-231 cell line results in greatly increased cancer cell invasion by elevating the expression of matrix metalloproteinase 9 (MMP9) and MMP13, both of which have been causally linked to the invasion and progression of numerous human solid tumors (22). In colon cancer, FoxO3a synergizes with beta-catenin to promote cell metastasis while conferring resistance to FoxO3a-induced apoptosis (23). These lines of evidence have challenged the previously defined role of FoxO3a in tumor progression. The role of FoxO3a is disputable in glioma, as the protein was revealed to mediate slow proliferation in GSCs (24) and however implied its involvement in tumor progression (25). 


\section{Materials and methods}

Cell lines and cell culture. The glioma cell line U251 was obtained from the Cell Bank of the Chinese Academy of Sciences (Shanghai, China). U87, A172 and T98g cell lines were gifts from the Neuroscience Institute of Soochow University. The normal human astrocyte 1800 cell line (HA1800) was obtained from the American Type Culture Collection (Manassas, VA, USA). All cells were cultured at $37^{\circ} \mathrm{C}$ in a humidified atmosphere containing $5 \% \mathrm{CO}_{2}$ and maintained in Dulbecco's modified Eagle's medium (DMEM; Gibco, Paisley, UK) supplemented with $10 \%$ heat inactivated fetal bovine serum (FBS; Gibco, Carlsbad, CA, USA), $100 \mu \mathrm{g} / \mathrm{ml}$ streptomycin and $1 \mathrm{U} / \mathrm{ml}$ penicillin.

Establishment of stable cell lines. DNA oligos designed by the BLOCK-iT RNAi designer (Invitrogen) encoding human FoxO3a-short hairpin RNAs [5'-GCTCTTGGTGGA TCATCAA-3' (FoxO3a-knockdown1) and 5'-GCATGTTCA ATGGGAGCTTGGA-3' (FoxO3a-knockdown2)] were synthesized and cloned into the pHY-LV-KD1.1 (Stored in our laboratory) vector to generate pHY-FoxO3a-KD1 and pHYFoxO3a-KD2. A vector expressing shRNA targeted against an irrelevant sequence (shRNA-NC) was used as a negative control. The full-length human FoxO3a cDNA was purchased from Open Biosystems and sub-cloned into the PHY-LV-OE1.6 vector (Stored in our laboratory). The recombined vector expressing FoxO3a was designated pHY-FoxO3a-OE. The Trans-Lentiviral Packaging System and Vira Power Lentiviral Expression System (Invitrogen) were used to produce shRNA and overexpress lentiviruses, respectively. shRNA lentiviruses were used to silence FoxO3a expression in U251 cells, while lentiviruses for overexpression were transduced into U87 cells. Stable cells, including FoxO3a-KD1 and FoxO3a-KD2 (both of which are U251 parental cell line knockdown derivatives), and FoxO3a-OE (U87 cells stably expressing FoxO3a), were generated using one week of puromycin selection.

Cell proliferation. Cell counts were determined using Cell Counting Kit-8 (Dojindo, Japan). A total of $1 \times 10^{3}$ GBM cells were plated onto 96-well culture plates in triplicate, and cell growth was determined daily for 5 days using a tetrazolium salt-based colorimetric assay (Dojindo Molecular Technologies) according to the manufacturer's protocol. Absorbance was measured at $450 \mathrm{~nm}$. Three independent experiments were performed.

Transwell invasion assay. For the Transwell assay, $2 \times 10^{4}$ stable cells were plated into 24-well Boyden chambers (Corning Costar, Cambridge, MA, USA) with an $8-\mu \mathrm{m}$ pore polycarbonate membrane coated with $30 \mu \mathrm{g}$ of Matrigel (BD Biosciences, San Jose, CA, USA). Cells were plated in the upper chamber with $200 \mu \mathrm{l}$ of serum-free medium, and medium containing $20 \%$ FBS was added to the lower chamber to serve as a chemoattractant. After $36 \mathrm{~h}$, the cells were washed 3 times with PBS. Non-invasive cells were removed from the upper well using cotton swabs, and the invasive cells were then fixed with paraformaldehyde for $15 \mathrm{~min}$, air-dried, and stained with $0.1 \%$ crystal violet for $15 \mathrm{~min}$. The cells were imaged with a digital camera.
Real-time PCR. Total RNA was extracted from stable cell lines and GBM parental cell lines. cDNA was prepared using $1 \mu \mathrm{g}$ of total RNA from each sample and using specific primers (Applied Biosystems). Six nanograms of cDNA were then used for real-time PCR analysis in a final reaction volume of $20 \mu 1$. Samples were analyzed in triplicate and statistical analysis was performed using the t-test.

Western blots. Total protein was extracted and separated by gel electrophoresis. Protein was then transferred to nitrocellulose membranes and probed overnight using the appropriate primary antibodies. The antibodies used were MMP1, MMP2, MMP3, MMP9 and MMP13 (Santa Cruz), cyclin D1 (CST, 2926), p21 (abcam, ab7960) and p27 (CST, 2552). Nuclear and cytoplasmic fractions of total protein were separated using NE-PER Nuclear and Cytoplasmic Extraction Reagent (Thermo Scientific, Rockford, IL, USA) and were then subjected to western blot analysis.

Statistical analysis. The data shown in the graphs represent the mean values $\pm \mathrm{SD}$ of three independent experiments. The difference among groups was determined by ANOVA, and comparison between two groups was analyzed by Student's t-test. A value of $\mathrm{P}<0.05$ was considered statistically significant.

\section{Results}

Expression and subcellular localization of FoxO3a in U87 and U251 cells. To investigate the expression pattern of FoxO3a in glioma cell lines, we employed western blotting to detect the protein level of FoxO3a. It was observed that among the four carcinoma cell lines (U87, U251, A172, and T98g), the FoxO3a levels in A172 and U87 were lower than in U251 and T98g (Fig. 1A). This finding is partially similar to that observed in a previous study (25). To explore the role of FoxO3a in glioma cell lines, U87 and U251 cells were selected for further investigation because not only did the two cell lines display a relatively inverse FoxO3a expression pattern, but they are also reliable glioma cell models that are known to mimic the salient features of human GBM in vitro. As FoxO3a transcriptional activity requires its nuclear accumulation, we detected its protein distribution in the nucleus and cytoplasm. Western blot analysis showed that a relatively high proportion of overall protein (nucleus: cytoplasm) accumulated in the nuclear fraction (Fig. 1B and C).

Effect of FoxO3a on proliferation. Because of the differential expression pattern of FoxO3a in U251 and U87 cells, we established stable cell lines containing FoxO3a overexpression and knockdown in the corresponding parental cell lines using a lentivirus-mediated approach. Stable transfectants were obtained through puromycin selection. In parallel cohorts of two clones, designated FoxO3a-KD1 and FoxO3a-KD2, we observed that knockdown of FoxO3a reduced U251 proliferation and that FoxO3a-KD1 had effects on cell proliferation despite better silencing (Fig. 2B). By contrast, increasing FoxO3a expression in U87 cells, which have relatively low levels of endogenous FoxO3a, promoted cell proliferation (Fig. 2A). To further confirm that FoxO3a promoted cell proliferation in these two cell models, we 
A

HA1800 U87 U251 A172 T98G

FoxO3a

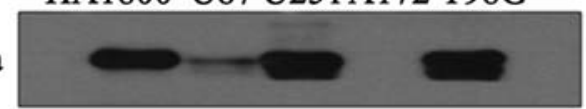

Actin

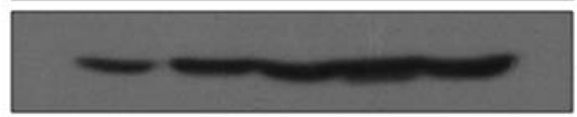

B

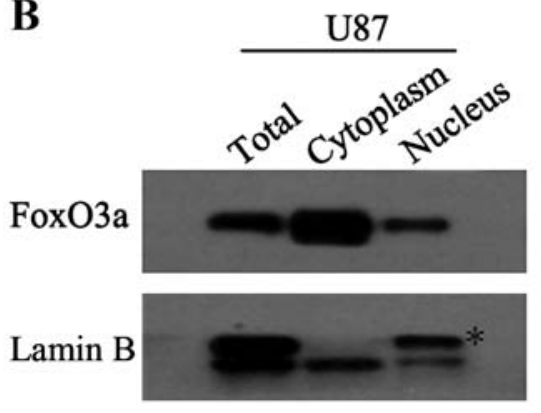

C $\mathrm{U} 251$

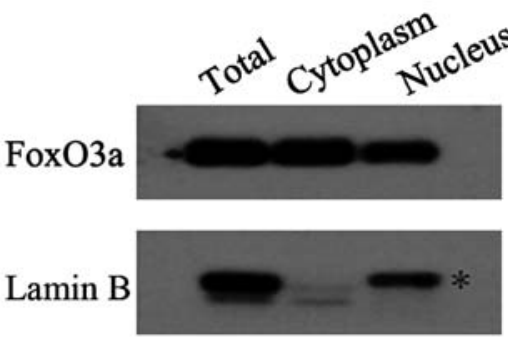

Figure 1. Expression of FoxO3a in U87 and U251 glioma cell lines. (A) Western blot analysis showing the levels of FOXO3a in human glioma cell lines (U87, U251, A172 and T98g cells) and the astrocyte 1800 cell line (HA1800). As a loading control, $\beta$-actin was also detected in the same blot. (B and C) Western blot analysis of FoxO3a in the cytoplasmic and nuclear fractions of the two glioma cell lines indicated. Actin and Lamin B were examined as cytoplasmic and nuclear markers. Two asterisks denote authentic bands for Lamin B.

A

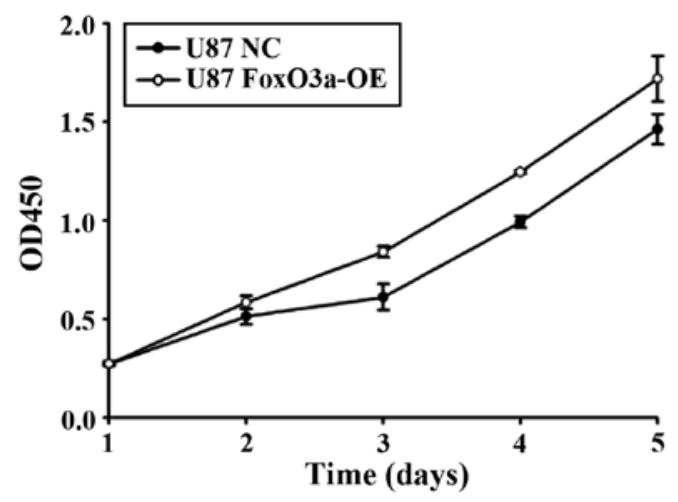

C

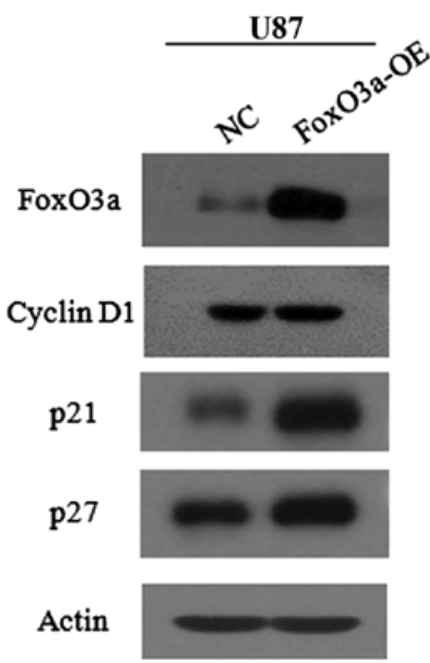

B

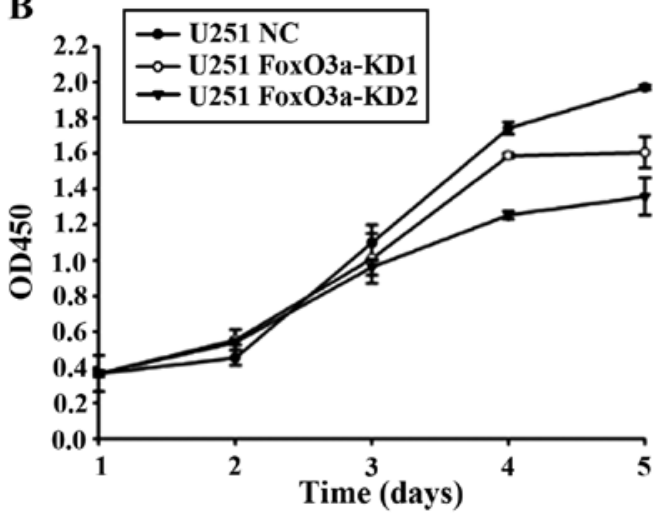

D

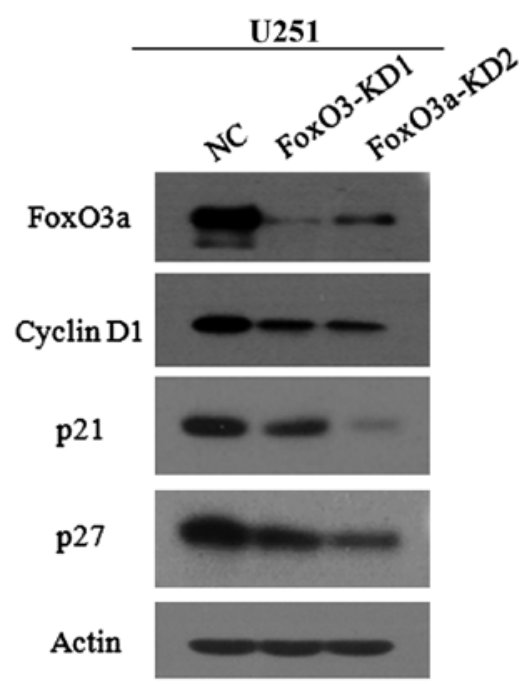

Figure 2. Effect of FoxO3a on glioma cell proliferation. (A) Dynamic changes in cell proliferation between U87 cells with ectopic expression of FoxO3a and those without (negative control, NC), as measured by the CCK8 assay from 1 to 5 days. (B) Dynamic changes in cell proliferation between U251 cells with FoxO3a knockdown and those without (negative control, NC), as measured by the CCK8 assay from 1 to 5 days. (C) Altered protein levels of FoxO3a target genes in cells with FoxO3a overexpression, as detected by western blotting. (D) Altered protein levels of FoxO3a target genes in cells with FoxO3a knockdown, as detected by western blotting. 
A

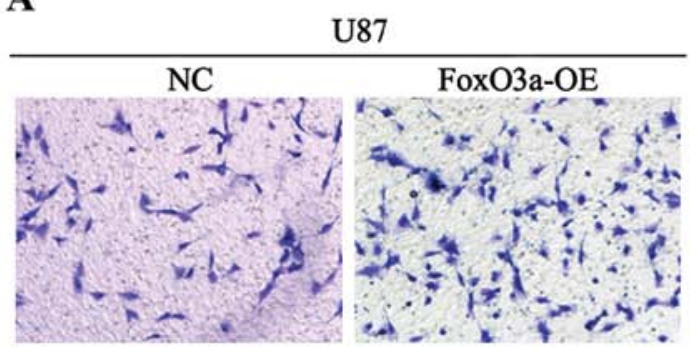

B

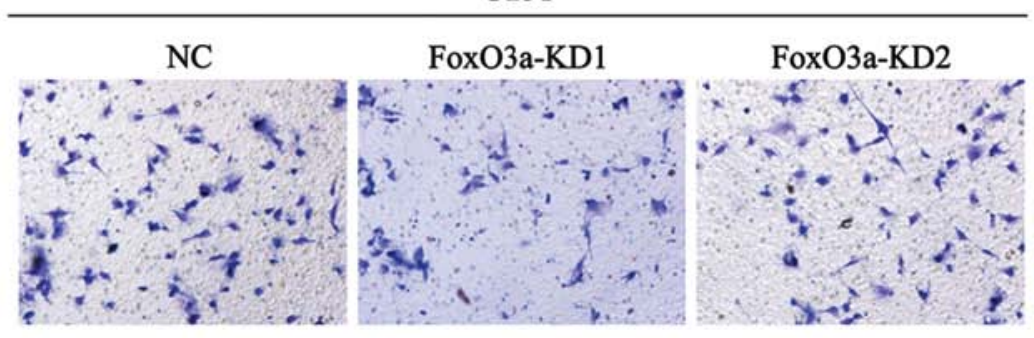

C

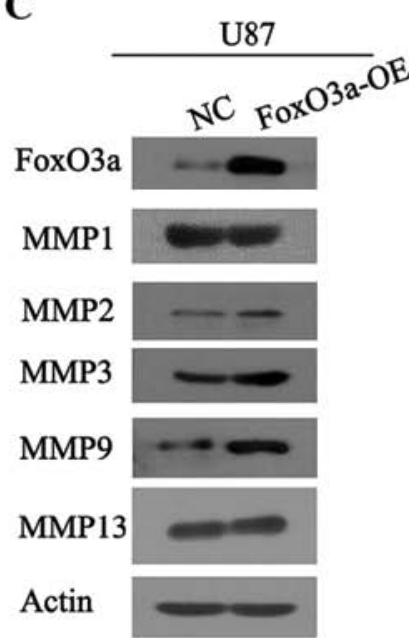

D

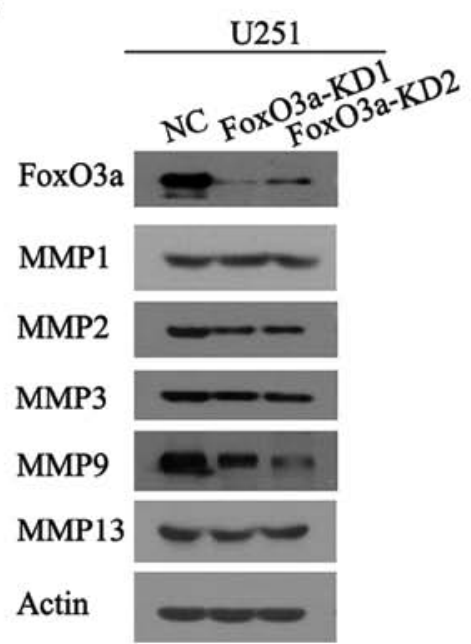

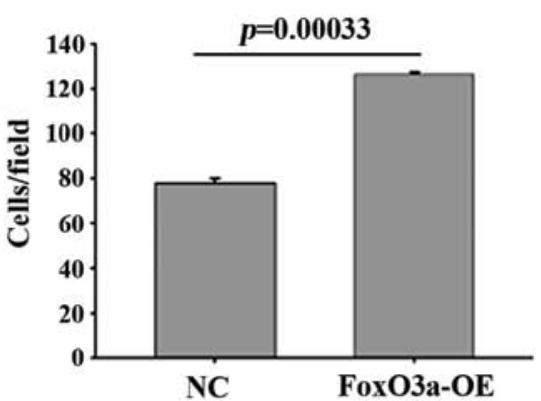

$-\mathrm{OE}$

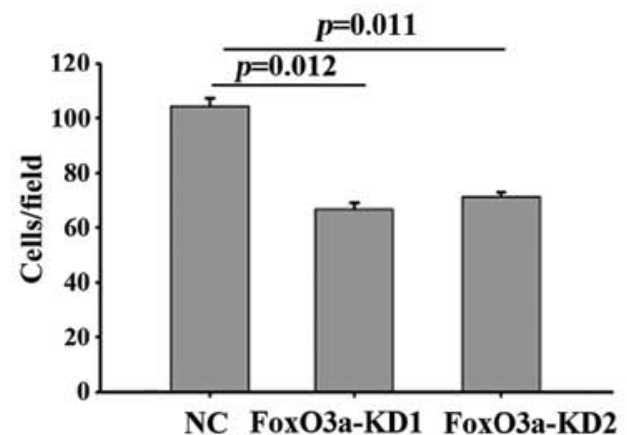

Figure 3. Effect of FoxO3a on glioma cell invasion. Cells were subjected to a Matrigel invasion chamber assay. (A) Altered invasion of U87 cells ectopically expressing FoxO3a was compared with that of a negative control. (B) Altered invasion of U251 cells with silencing of FoxO3a was compared with a negative control (NC). Altered expression of certain MMPs in cells with FoxO3a overexpression (C) and knockdown (D), respectively. The numbers of invaded cells are shown in the right panel. The data represent the mean $\pm \mathrm{SD}$ of three independent experiments. P-values were acquired using the $\mathrm{t}$-test. $\mathrm{P}$-values indicate statistical significance.

detected the expression of FoxO3a target genes associated with cell arrest and cell growth, including p27, p21 and cyclin D1. Silencing FoxO3a led to p27, p21 and cyclin D1 downregulation (Fig. 2D), whereas elevating FoxO3a expression upregulated these target genes (Fig. 2C). In effect, FoxO3a was able to promote cell proliferation, as determined by an in vitro proliferation assay, suggesting that FoxO3a has a dominant effect on cell growth over cell arrest. We then determined whether FoxO3a expression could trigger apoptosis. The findings from these stable glioma cells indicated that FoxO3a exerted little effect on apoptosis (data not shown).

Effect of FoxO3a on invasion of glioma cells. To study the role of FoxO3a in glioma cell invasion in vitro, we analyzed the invasion potential of the stably transfected cells expressing
FoxO3a using a matrigel invasion chamber assay. As shown in Fig. 3A, ectopic expression of FoxO3a in U87 cells led to a significant increase in invasion compared with the control of glioma cells. By contrast, knockdown of FoxO3a in U251 cells led to a marked decrease in invasion, as observed by the smaller number of cells penetrating through the chambers (Fig. 3B). The findings from the two cell models demonstrate that FoxO3a affects the invasion of glioma cells. Furthermore, we detected the expression of key MMP members associated with tumor metastasis. The results showed that FoxO3a overexpression induced significantly elevated expression of MMP9, while other well-defined candidates associated with metastasis, such as MMP2 and MMP13, did not show any marked increase (Fig. 3C). Furthermore, the U251 stable cell line FoxO3a-KD1 showed attenuated MMP9 protein levels 
U251

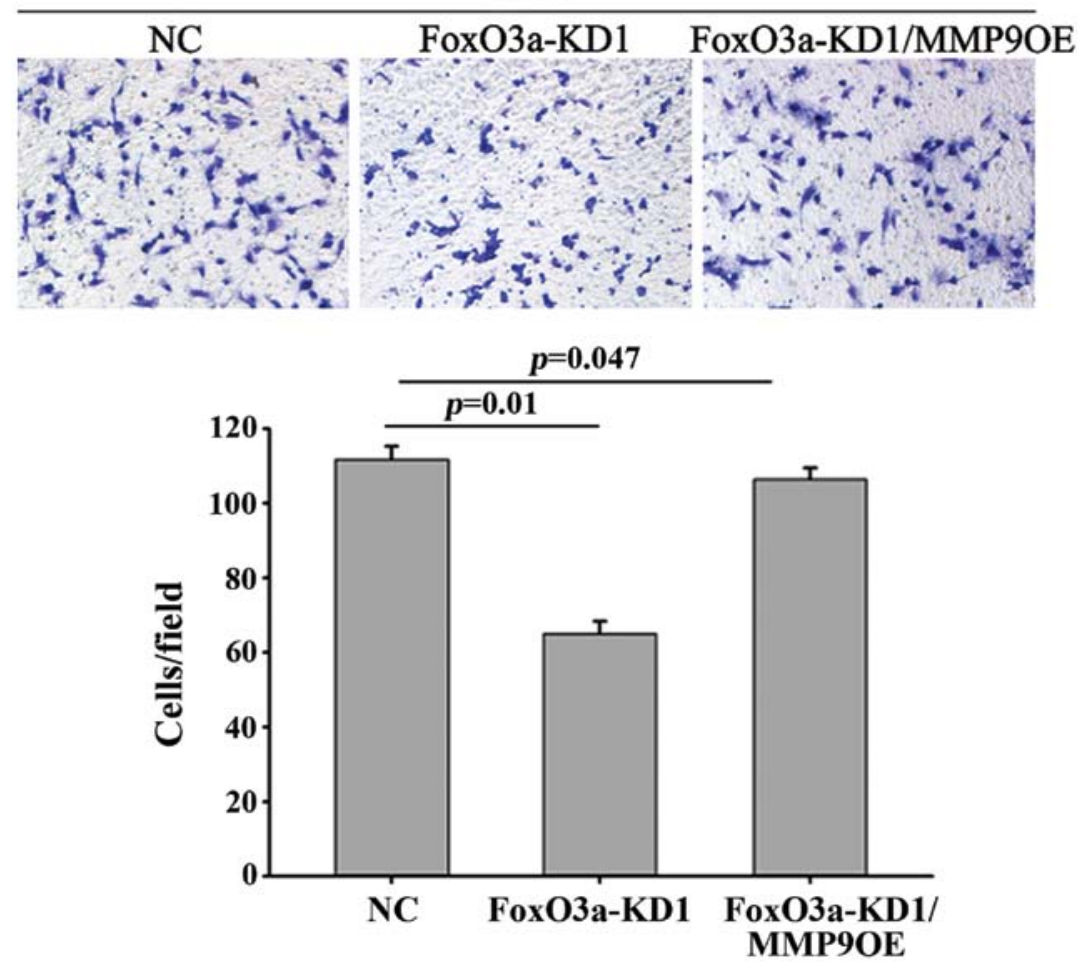

Figure 4. Effect of MMP9 overexpression on the invasive ability of U251 cells stably silencing FoxO3a. Comparison of cell invasion between parental U251 cells (NC), stable U251 cells (stably silencing FoxO3a, FoxO3a-KD1), and stable U251 cells following overexpression of MMP9 (FoxO3a/MMP9OE). The numbers of invaded cells are shown in the lower panel. P-values were acquired using the t-test. P-values indicate statistical significance. All results are typical of three independent experiments.

and decreased invasion (Fig. 3D). Following MMP9 overexpression, the impaired invasive ability mediated by FoxO3a depletion appeared to recover (Fig. 4).

\section{Discussion}

In the present study, we investigated the FoxO3a expression spectrum in several glioma cell lines and used the U251 and U87 cell lines to further investigate the role of FoxO3a in glioma cells. FoxO3a overexpression in U87 cells did not attenuate the cell proliferative capacity but instead increased cell proliferation. This finding is consistent with observations in U251 cells, where the silencing of FoxO3a caused a decrease in proliferation. Unlike most researchers, who claim that FoxO3a can trigger apoptosis, we did not observe that FoxO3a expression induced apoptosis in glioma cells (data not shown). Furthermore, we determined whether FoxO3a had an impact on cell invasion. It was observed that the downregulation of FoxO3a significantly inhibited the invasion of U251 cells, whereas the overexpression of FoxO3a enhanced the invasion of U87 cells. Following stable FoxO3a overexpression and knockdown in cells, we detected changes in the protein levels of MMPs. The results showed that FoxO3a manipulated MMP9 expression while having no effect on other MMPs. Overexpression of MMP9 in FoxO3a-KD1 cells restored the compromised invasive ability mediated by FoxO3a silencing, indicating that FoxO3a increases glioma cell invasion through the induction of MMP9 alone.

Despite technological advances in surgical and radiological techniques, malignant gliomas often recur within
1-2 $\mathrm{cm}$ of the original tumor site (2). Key characteristic features, such as the extensive infiltration of invasive cells into surrounding normal tissue, allow gliomas to evade surgical removal and radiation therapy. Despite the need for improved therapies, the molecular mechanisms responsible for invasion remain largely undefined. This encourages the investigation of many cancer-related genes, including FoxO3a. We observed that FoxO3a promoted the invasion of glioma cells, which is at odds with previous studies defining FoxO3a as a tumor suppressor. Nonetheless, several recent studies favor our findings demonstrating that FoxO3a participates in cell invasion: i) FoxO3a activation in breast cancer tissue was significantly associated with lymph node metastasis (8), ii) depletion of FoxO3a resulted in a significant decrease in the invasive migration of MDA-MB-435 cells (22) and iii) high levels of activated FoxO3a were present in cells with a high metastatic potential.

In addition, we observed that FoxO3a promoted cell invasion through the induction of MMP9 (Fig. 3C and D). MMP9, a key member of the MMP family, is linked to metastasis in a variety of cancer types (26). Overexpression of MMP9 is also associated with a high metastatic potential in rat osteosarcoma cell lines (27). Chemically-induced skin tumors exhibited high MMP9 expression only in invasive skin carcinomas (28). Increased expression of MMP9 has been found in anaplastic astrocytomas and GBM (29), and this increased expression is also correlated with the progression of highly malignant human gliomas in vivo. In MDA-MB-231 cells, it appears that MMP9 expression, combined with the expression of other MMP members, such as MMP2 and MMP13, could lead to 
cell invasion (22). However, we did not observe that FoxO3a induced the expression of MMP2 and MMP13, instead we only observed the induction of MMP9 (Fig. 3C and D).

Similar findings have been reported in studies of spontaneous murine breast carcinomas, in which researchers discovered that MMP9 was the only metalloproteinase whose expression correlated with the metastatic properties of a parental cell line derivative (30). Using AG1478, researchers observed significantly reduced MMP9 activity, but not MMP2 activity, and the reduced MMP9 activity correlated with a decrease in cell invasion (31). In addition, GBM patient specimens were shown to express high levels of MMP9 protein, but low levels of other MMPs, and MMP9 was present in human GBM tumor masses as well as in tumor cells diffusely invading adjacent brain tissue (31), suggesting that manipulation of the MMP9 protein level alone might render glioma cells capable or incapable of invasion. Our findings have validated the relationship between MMP9 and glioma cell invasion. Ectopic expression of MMP9 restored the invasive potential that would have otherwise been attenuated by the silencing of FoxO3a (Fig. 4), indicating that FoxO3a-induced MMP9 alone may play a critical role in cell invasion. However, the MMP9 promoter does not contain a FoxO3a DNA binding motif. This implies that FoxO3a may cooperate with a specific transcription factor of MMP9 (SMAD3/4 or NF- $\kappa \mathrm{B}$ ), thereby activating expression through the promoter, which lacks FOXO binding sites.

The effect of FoxO3a on cell proliferation and apoptosis was investigated, and our findings indicated that FoxO3a did not induce either cell death or cell cycle arrest. On the contrary, FoxO3a increased cell proliferation. FoxO3a target genes associated with cell proliferation and apoptosis were concurrently activated. Of note, we observed the upregulation of FASLG and HIPK3 (data not shown), two genes that are co-regulated by FoxO3a and beta-catenin in colon cancer (23). This finding provides a clue for further investigations exploring the unknown mechanisms that FoxO3a uses to override its own anti-proliferative function in glioma cells. For instance, we might test whether the coordinated mechanism of FoxO3a and beta-catenin that has been unraveled in colon cancer (23) has a role in gliomas.

Few studies have correlated FoxO3a with the promotion of cancer progression. On the contrary, most studies have defined FoxO3a as a tumor suppressor. Herein, by providing compelling evidence with respect to cell proliferation and invasion, we propose that in glioma cells, FoxO3a plays a part in cancer progression.

\section{Acknowledgements}

We are grateful to the National Natural Science Foundation of China (Grant \# 31260225, 81660502) and Natural Science Fund for Colleges and Universities in Jiangsu Province (16KJB320015) for funding this research.

\section{References}

1. Ohgaki H: Genetic pathways to glioblastomas. Neuropathology 25: 1-7, 2005

2. Hou LC, Veeravagu A, Hsu AR and Tse VC: Recurrent glioblastoma multiforme: A review of natural history and management options. Neurosurg Focus 20: E5, 2006.
3. Friedl P and Alexander S: Cancer invasion and the microenvironment: Plasticity and reciprocity. Cell 147: 992-1009, 2011.

4. Tonn JC and Goldbrunner R: Mechanisms of glioma cell invasion. In: Local Therapies for Glioma Present Status and Future Developments. Springer, pp163-167, 2003. doi: org/10.1007/9783-7091-6090-9_22

5. Fu Z and Tindall DJ: FOXOs, cancer and regulation of apoptosis. Oncogene 27: 2312-2319, 2008

6. Hu M, Lee D, Xia W, Xia W, Golfman LS, Ou-Yang F, Yang JY, Zou Y, Bao S, Hanada N, et al: IkappaB kinase promotes tumourigenesis through inhibition of Forkhead transcription factor FOXO3a. Cell 117: 225-237, 2004.

7. Yang J-Y, Chang C-J, Xia W, Wang Y, Wong KK, Engelman JA, Du Y, Andreeff M, Hortobagyi GN and Hung MC: Activation of FOXO3a is sufficient to reverse mitogen-activated protein/extracellular signal-regulated kinase kinase inhibitor chemoresistance in human cancer. Cancer Res 70: 4709-4718, 2010.

8. Chen J, Gomes AR, Monteiro LJ, Wong SY, Wu LH, Ng TT, Karadedou CT, Millour J, Ip YC, Cheung YN, et al: Constitutively nuclear FOXO3a localization predicts poor survival and promotes Akt phosphorylation in breast cancer. PLoS One 5: e12293, 2010.

9. Lam M, Carmichael AR and Griffiths HR: An aqueous extract of Fagonia cretica induces DNA damage, cell cycle arrest and apoptosis in breast cancer cells via FOXO3a and p53 expression. PLoS One 7: e40152, 2012.

10. Shukla S, Bhaskaran N, Maclennan GT and Gupta S: Deregulation of FoxO3a accelerates prostate cancer progression in TRAMP mice. Prostate 73: 1507-1517, 2013.

11. Shukla S, Bhaskaran N, Babcook MA, Fu P, Maclennan GT and Gupta S: Apigenin inhibits prostate cancer progression in TRAMP mice via targeting PI3K/Akt/FoxO pathway. Carcinogenesis 35: 452-460, 2014.

12. Chen Q, Ganapathy S, Singh KP, Shankar S and Srivastava RK: Resveratrol induces growth arrest and apoptosis through activation of FOXO transcription factors in prostate cancer cells. PLoS One 5: e15288, 2010.

13. Sunayama J, Sato A, Matsuda K, Tachibana K, Watanabe E, Seino S, Suzuki K, Narita Y, Shibui S, Sakurada K, et al: FoxO3a functions as a key integrator of cellular signals that control glioblastoma stem-like cell differentiation and tumorigenicity. Stem Cells 29: 1327-1337, 2011.

14. Wang W, Li N-N, Du Y, Lv F-F and Lin G-Q: FoxO3a and nilotinib-induced erythroid differentiation of CML-BC cells. Leuk Res 37: 1309-1314, 2013.

15. Ruvolo PP: The Herculean task of killing cancer cells: Suppression of FOXO3A in acute leukemia involves a hydra of multiple survival kinases. Cell Cycle 11: 2589-2589, 2012.

16. Kops GJ, Medema RH, Glassford J, Essers MA, Dijkers PF, Coffer PJ, Lam EW and Burgering BM: Control of cell cycle exit and entry by protein kinase B-regulated forkhead transcription factors. Mol Cell Biol 22: 2025-2036, 2002.

17. Hauck L, Harms C, Grothe D, An J, Gertz K, Kronenberg G, Dietz R, Endres M and von Harsdorf R: Critical role for FoxO3adependent regulation of $\mathrm{p} 21 \mathrm{CIP} 1 / \mathrm{WAF} 1$ in response to statin signaling in cardiac myocytes. Circ Res 100: 50-60, 2007.

18. Miyauchi H, Minamino T, Tateno K, Kunieda T, Toko H and Komuro I: Akt negatively regulates the in vitro lifespan of human endothelial cells via a p53/p21-dependent pathway. EMBO J 23: 212-220, 2004.

19. Rathbone CR, Booth FW and Lees SJ: FoxO3a preferentially induces $\mathrm{p} 27 \mathrm{Kip} 1$ expression while impairing muscle precursor cell-cycle progression. Muscle Nerve 37: 84-89, 2008.

20. Roy SK, Chen Q, Fu J, Shankar S and Srivastava RK: Resveratrol inhibits growth of orthotopic pancreatic tumors through activation of FOXO transcription factors. PLoS One 6: e25166, 2011.

21. Obexer P, Geiger K, Ambros PF, Meister B and Ausserlechner MJ: FKHRL1-mediated expression of Noxa and Bim induces apoptosis via the mitochondria in neuroblastoma cells. Cell Death Differ 14: 534-547, 2007.

22. Storz P, Döppler H, Copland JA, Simpson KJ and Toker A: FOXO3a promotes tumor cell invasion through the induction of matrix metalloproteinases. Mol Cell Biol 29: 4906-4917, 2009.

23. Tenbaum SP, Ordóñez-Morán P, Puig I, Chicote I, Arqués O, Landolfi S, Fernández Y, Herance JR, Gispert JD, Mendizabal L, et al: $\beta$-catenin confers resistance to PI3K and AKT inhibitors and subverts FOXO3a to promote metastasis in colon cancer. Nat Med 18: 892-901, 2012. 
24. Osuka S, Sampetrean O, Shimizu T, Saga I, Onishi N, Sugihara E, Okubo J, Fujita S, Takano S, Matsumura A, et al: IGF1 receptor signaling regulates adaptive radioprotection in glioma stem cells. Stem Cells 31: 627-640, 2013.

25. Ling N, Gu J, Lei Z, Li M, Zhao J, Zhang HT and Li X: microRNA-155 regulates cell proliferation and invasion by targeting FOXO3a in glioma. Oncol Rep 30: 2111-2118, 2013

26. Deryugina EI and Quigley JP: Matrix metalloproteinases and tumor metastasis. Cancer Metastasis Rev 25: 9-34, 2006.

27. Troussard AA, Costello P, Yoganathan TN, Kumagai S Roskelley CD and Dedhar S: The integrin linked kinase (ILK) induces an invasive phenotype via AP-1 transcription factordependent upregulation of matrix metalloproteinase 9 (MMP-9). Oncogene 19: 5444-5452, 2000.

28. Kupferman ME, Fini ME, Muller WJ, Weber R, Cheng Y and Muschel RJ: Matrix metalloproteinase 9 promoter activity is induced coincident with invasion during tumor progression. Am J Pathol 157: 1777-1783, 2000.
29. Rao JS, Yamamoto M, Mohaman S, Gokaslan ZL, Fuller GN, Stetler-Stevenson WG, Rao VH, Liotta LA, Nicolson GL and Sawaya RE: Expression and localization of $92 \mathrm{kDa}$ type IV collagenase/gelatinase B (MMP-9) in human gliomas. Clin Exp Metastasis 14: 12-18, 1996.

30. Yang J, Mani SA, Donaher JL, Ramaswamy S, Itzykson RA, Come C, Savagner P, Gitelman I, Richardson A and Weinberg RA: Twist, a master regulator of morphogenesis, plays an essential role in tumor metastasis. Cell 117: 927-939, 2004.

31. Zhao Y, Xiao A, diPierro CG, Carpenter JE, Abdel-Fattah R, Redpath GT, Lopes MB and Hussaini IM: An extensive invasive intracranial human glioblastoma xenograft model: Role of high level matrix metalloproteinase 9. Am J Pathol 176: 3032-3049, 2010. 